\title{
Amorphization in crystalline tellurium by femtosecond pulses
}

\author{
Yu-Hsiang Cheng ${ }^{1, *}$, Samuel Teitelbaum ${ }^{2}$, Frank $\mathrm{Gao}^{2}$, and Keith Nelson ${ }^{2}$ \\ ${ }^{1}$ Department of Electrical Engineering and Computer Science, Massachusetts Institute of Technology, \\ Cambridge, MA 02139, USA \\ ${ }^{2}$ Department of Chemistry, Massachusetts Institute of Technology, Cambridge, MA 02139, USA
}

\begin{abstract}
Crystalline tellurium undergoes amorphization after irradiation with strong femtosecond pulses. Steady-state reflectivity and single-shot transient reflectivity studies suggest the amorphization is due to thermal melting rather than non-thermal switching.
\end{abstract}

\section{Introduction}

Tellurium is an example of a phase-change material whose crystalline and amorphous phases can be switched reversibly by optical illumination when kept below its glass transition temperature $(\sim 285 \mathrm{~K})$ [1]. It is generally believed that amorphization follows melting of the material but it is still unclear whether the melting is thermal (due to high lattice temperature) or non-thermal (due to high carrier density) under irradiation of femtosecond pulses [2]. In this paper, we study the phase transition at low temperatures (down to $80 \mathrm{~K}$ ), so a higher carrier density can be optically excited before the lattice temperature reaches the melting point. For all fluences applied below the ablation threshold, we believe the amorphization is the result of thermal melting.

\section{Experiment}

The sample was a 250 -nm-thick tellurium polycrystalline thin film, sputtered onto a glass slide. The sample was kept in a liquid nitrogen flow cryostat to control its temperature. The details of the single-shot pump-pump-probe setup can be found in the reference [3]. The laser source was a Ti:sapphire regenerative amplifier with a 2 -mJ pulse energy and 60 -fs pulse width at a repetition rate of $1 \mathrm{kHz}$. The majority of the pulse energy was sent to a Michelson interferometer to create double excitations. The rest of the energy acted as the probe which passed through two crossed echelons to form 400 beamlets separated by $23 \mathrm{fs}$ steps. By comparing the probe signal reflected from the sample with pump and without pump, a transient reflectivity trace covering 9.2 ps could be acquired with a single laser shot. In addition, the change in the steady-state reflectivity was also monitored shot-byshot.

\footnotetext{
*Corresponding author: yuhsiang@mit.edu
} 


\section{Results}

After optical illumination above the amorphization threshold $\left(\sim 7 \mathrm{~mJ} / \mathrm{cm}^{2}\right.$ at $\left.80 \mathrm{~K}\right)$, part of the film near the surface was turned amorphous and the steady-state reflectivity of the polycrystalline tellurium sample decreased. As the volume of amorphized material increased with photon density, the change in steady-state reflectivity increased linearly with pump fluence. The amorphization was mostly complete with a single pump shot and the following shots resulted in only a minor increase in the volume fraction of the amorphous phase. The threshold and saturation behaviours are common features of photoinduced phase transitions due to the competition of two coexisting phases and the spatial profile of the optical excitation. When exposed with fluences above the ablation threshold $\left(\sim 12 \mathrm{~mJ} / \mathrm{cm}^{2}\right.$ at $80 \mathrm{~K})$, the steady-state reflectivity did not reach the saturation value within 10 shots, but continued to drop for a few hundred shots. Unlike the amorphized regions, the damaged regions did not return to the initial crystalline phase after being warmed to room temperature.

As shown in Fig. 1(a), the amorphization process was sensitive to the initial temperature of the sample. Exposed to the same pump pulses, the change in the steady-state reflectivity varied linearly with the initial temperature. The amorphization threshold decreased from $7 \mathrm{~mJ} / \mathrm{cm}^{2}$ at $80 \mathrm{~K}$ to $\sim 6 \mathrm{~mJ} / \mathrm{cm}^{2}$ at $240 \mathrm{~K}$. Such behaviour is consistent with amorphization as a result of thermal melting.

To further determine the mechanism of the melting, we varied the temporal profile of the optical excitation. First, we stretched the pump pulse and found the amorphization process was not very sensitive to the pulse width, shown in Fig. 1(b). Secondly, we excited the sample with two identical but weaker pump pulses. As shown in Fig. 1(c), we found similar degrees of amorphization still occurred when the two pumps were separated by 100 ps, which is sufficient for the majority of excited carriers near the surface to diffuse into the bulk region. Therefore, this phase transition was likely a thermal process rather than the non-thermal melting induced by high carrier density. In the case of homogenous melting, the lattice temperature was ramped much higher than the melting point $(723 \mathrm{~K})$ in order to overcome the heat of fusion within a few picoseconds [2] and then entered the amorphous phase after fast quenching.

In the transient reflectivity traces, we observed clear coherent $A_{1}$ phonon oscillations due to displacement excitation by femtosecond pulses. In the low fluence regime, as the pump fluence increased, the phonon amplitude increased, the phonon damping increased, and the phonon frequency decreased due to electronic bond softening. When excited above the amorphization threshold, more than one phonon cycle were still observable in the single-shot transient reflectivity trace, shown in Fig. 1(d). Therefore, the lattice was not melted within the first picosecond but did so later on.

When excited above the amorphization threshold, the transient reflectivity trace changed shot-by-shot, similar to the steady-state reflectivity. Only the first-shot response represented the photoexcited dynamics of the crystalline phase. After the first shot, part of the sample near the surface was turned amorphous so the observed trace excited by later shots were the result of a mixed response of the photoexcited amorphous phase and the underlying crystalline phase. Since the reflectivity and absorption coefficients near $1.5 \mathrm{eV}$ are both noticeably lower in the amorphous state than in the crystalline state, the thermal gradient of the excited sample depends on its degree of amorphization. Therefore, the relaxation constant of the trace induced by the first pump shot when the initial state was crystalline was quite different from those excited by later pump pulses where part of the sample was initially amorphous. 
In addition, we performed transient reflectivity measurement on the photo-darkened region at lower fluences. When the original photo-darkening pump was not too strong, the coherent phonon oscillation was suppressed but still could be seen, indicating that we were probing a mixture of crystalline and amorphous states. With stronger original pumps, the whole probed volume was turned amorphous, and the phonon oscillation disappeared.

When the sample was kept above the glass transition temperature but below the melting point, the amorphous phase quickly relaxed back into the crystalline phase. For example, at room temperature, the photoinduced amorphous spot disappeared within 1 second. By measuring the spontaneous recrystallization rate at various temperatures and fitting to the Arrhenius equation, we estimated the activation energy to be $0.82 \mathrm{eV}$.

The recrystallization at low temperatures can be done by heating using femtosecond pulses with moderate fluences $\left(<7 \mathrm{~mJ} / \mathrm{cm}^{2}\right)$. Although amorphization can be achieved by one powerful pulse, recrystallization usually requires multiple weak pulses. At $80 \mathrm{~K}$, after exposed to 50 pulses with fluence of $6 \mathrm{~mJ} / \mathrm{cm}^{2}$, most amorphous regions can be returned to the crystalline phase. With $2 \mathrm{~mJ} / \mathrm{cm}^{2}$ pulses, the recovery took more than 4000 shots. In the transient reflectivity traces, we observed the shot-by-shot recovery of coherent phonons.
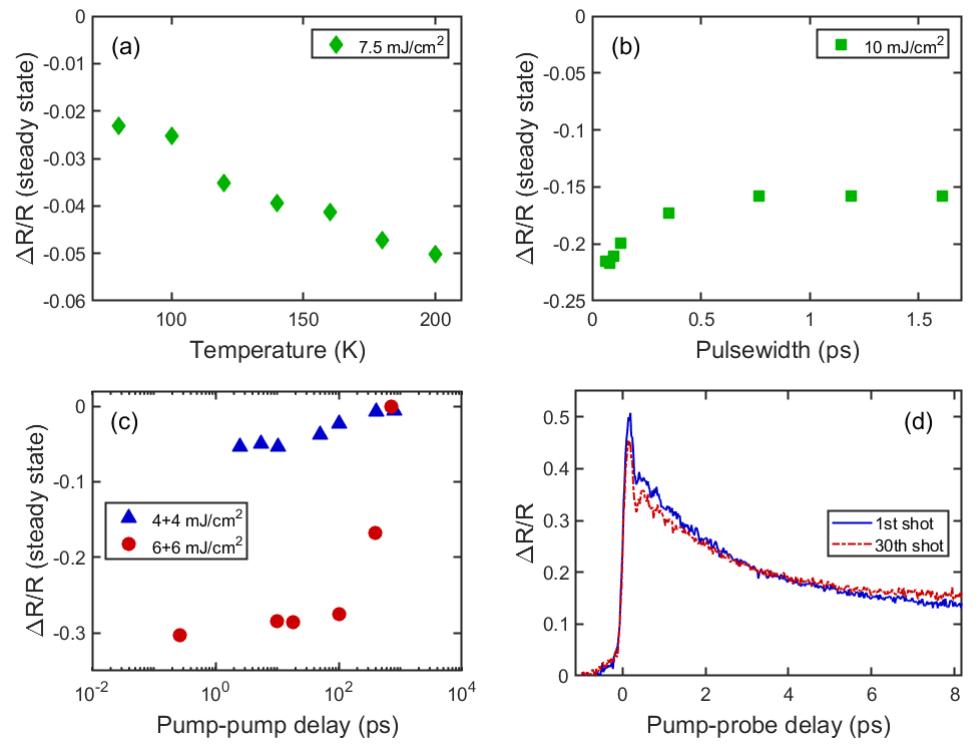

Fig. 1. The change in steady-state reflectivity under single-pump excitation with (a) different initial temperatures and (b) different pump pulse widths and (c) under double-pump excitation with various pump-pump delays. (d) The single-shot transient reflectivity trace under single-pump excitation with fluence of $7.5 \mathrm{~mJ} / \mathrm{cm}^{2}$. (b), (c), and (d) were measured at $80 \mathrm{~K}$.

\section{References}

1. P. C. Clemens, Appl. Opt. 22, 3165 (1983).

2. S. I. Ashitkov, M. B. Agranat, P. S. Kondratenko, S. I. Anisimov, V. E. Fortov, V. V. Temnov, K. Sokolowski-Tinten, B. Rethfeld, P. Zhou, and D. Linde, J. Exp. Theor. Phys. Lett. 76, 461 (2002).

3. Y.-H. Cheng, F. Y. Gao, S. W. Teitelbaum, and K. A. Nelson, Phys. Rev. B 96, 134302 (2017). 